\title{
VARIETIES WITH AMPLE TANGENT SHEAVES
}

\author{
PHILIP SIEDER
}

\begin{abstract}
AвSTRACT. This paper generalises Mori's famous theorem about "Projective manifolds with ample tangent bundles" [Mor79] to normal projective varieties in the following way:

A normal projective variety over $\mathbb{C}$ with ample tangent sheaf is isomorphic to the complex projective space.
\end{abstract}

\section{INTRODUCTION}

In this paper we give a proof for the following theorem.

Main Theorem. A normal projective variety over $\mathbb{C}$ with ample tangent sheaf is isomorphic to the projective space.

We work over the field of complex numbers $\mathbb{C}$. Besides that restriction, the theorem is a generalisation to singular varieties of Mori's famous result.

Theorem ([Mor79]]). An $n$-dimensional projective manifold $X$ over an algebraically closed field $\mathbb{K}$ with ample tangent bundle is isomorphic to the projective space $\mathbb{P}_{\mathbb{K}}^{n}$.

Mori's work has been generalised over the years in various ways, for example by Andreatta and Wiśniewski [AW01]: For $X$ being $\mathbb{P}_{n}$ it suffices that $\mathcal{T}_{X}$ contains an ample subbundle. This has been altered by Aprodu, Kebekus and Peternell [AKP08, Section 4]. They add the assumption that $X$ has Picard number 1 , but an ample subsheaf (not necessarily locally free) of $\mathcal{T}_{X}$ then induces $X \simeq \mathbb{P}_{n}$. Generalising those results, Liu [Liu16] recently showed that $X$ is already the projective space if $\mathcal{T}_{X}$ contains an ample subsheaf (again not necessarily locally free). Kebekus [Keb02] even characterises $\mathbb{P}_{n}$ only by using the anticanonical degree of all rational curves being greater than $n$. All these efforts, besides Ballico's article [Bal93], keep the preliminary that $X$ is smooth. Ballico's paper on the other hand treats mainly positive characteristic, as he requires the tangent sheaf to be locally free. Which, the Zariski-Lipman conjecture suggests, is most likely never the case over the complex numbers, if $X$ is singular. 
Outline of our proof. We consider a special desingularisation $\hat{X}$ of the given variety $X$ of dimension $\geq 2$ (normal curves are smooth) and prove that $\hat{X}$ is the projective space. As $\mathbb{P}_{n}$ is minimal, $X$ itself is already the projective space. To show that $\hat{X}$ is the projective space, we combine two strong results.

First, we relate $\mathcal{T}_{X}$ to $\mathcal{T}_{\hat{X}}$ : For a suitable desingularisation $\pi: \hat{X} \rightarrow X$, there is a morphism $f: \pi^{*} \mathcal{T}_{X} \rightarrow \mathcal{T}_{\hat{X}}$ that is an isomorphism outside $\pi^{-1}(\operatorname{Sing}(X))$ (Theorem 3.2).

Secondly, we use a corollary given by Cho, Miyaoka and Shepherd-Barron [CMSB02, Corollary 0.4 (11)] that Kebekus [Keb02] later proved directly (although he claims a weaker result): A uniruled manifold $\hat{X}$ is isomorphic to the projective space, if the anticanonical degree $-K_{\hat{X}} . \hat{C}$ is greater or equal $n+1$ for all rational curves $\hat{C}$ through a general point $p$. The uniruledness of $\hat{X}$ follows from the negativity of $K_{\hat{X}}$ and the anticanonical degree is calculated using the splitting of $\left.\mathcal{T}_{\hat{X}}\right|_{\hat{C}}$ on the normalisation of $\hat{C}$ (Lemma 3.3). Hence $\hat{X} \simeq \mathbb{P}_{n} \simeq X$.

\section{Preliminaries}

Let us first recall the definition of the tangent sheaf for a proper variety, as it is a central term in this paper.

Definition 2.1 (tangent sheaf). Let $X$ be a algebraic variety, then its tangent sheaf $\mathcal{T}_{X}:=\mathcal{H o m}\left(\Omega_{X}^{1}, \mathcal{O}_{X}\right)$ is the dual of the cotangent sheaf.

We want to work on a desingularisation $\hat{X}$ of the normal variety $X$, so we have to connect $\mathcal{T}_{X}$ with $\mathcal{T}_{\hat{X}}$ :

Theorem 2.2. Let $X$ be a normal projective variety with tangent sheaf $\mathcal{T}_{X}$. Then there is a desingularisation $\pi: \hat{X} \rightarrow X$ and an $\mathcal{O}_{X}$-module isomorphism

$$
\mathcal{T}_{X} \rightarrow \pi_{*} \mathcal{T}_{\hat{X}}
$$

Proof. Graf and Kovács [GK14, Theorem 4.2] state that there is a resolution $\pi: \hat{X} \rightarrow X$ such that $\pi_{*} \mathcal{T}_{\hat{X}}$ is reflexive. The sheaves $\mathcal{T}_{X}$ and $\pi_{*} \mathcal{T}_{\hat{X}}$ are reflexive, $X$ is normal and $\pi$ is an isomorphism outside the preimage of a set of codimension 2. Thus we obtain an isomorphism $\mathcal{T}_{X} \rightarrow \pi_{*} \mathcal{T}_{\hat{X}}$.

Remark. For a more thorough understanding of the map $\mathcal{T}_{X} \rightarrow \pi_{*} \mathcal{T}_{\hat{X}}$ and the resolution $\pi$, see the paper of Greb, Kebekus and Kovács [GKK10, Section 4]. 
The most cited definition for ample sheaves is in Ancona's paper [Anc82]. He defines ampleness and provides some equivalent characterisations, but gives very few properties. Kubota [Kub70] on the other hand works over graded $\mathcal{O}_{X}$-modules and gives some properties, but does not use the most modern language.

So we recall a definition and the most important properties we use throughout this work.

Definition 2.3 (ample sheaf). Let $X$ be a proper algebraic variety and $\mathcal{E}$ a coherent sheaf on $X$. Then we say $\mathcal{E}$ is ample if for every coherent sheaf $\mathcal{F}$ on $X$ there exists an $n=n(\mathcal{F})$ such that $\mathcal{F} \otimes S^{m} \mathcal{E}$ is globally generated for $m \geq n$.

Remark. Other characterisations of ampleness can be found in [Anc82]. Note that an ample sheaf, unlike an ample vector bundle, on a proper variety $X$ does not yield that its support is projective, but only Moishezon [GPR94, Remark p. 244].

The following properties can be found in Debarre's paper [Deb06, Section 2] or the proof in the vector bundle case (as in [Laz04]) carries over to coherent sheaves:

Proposition 2.4. Let $X$ and $Y$ be normal projective varieties, $f: Y \rightarrow X a$ finite morphism, $\mathcal{E}, \mathcal{E}_{1}$ and $\mathcal{E}_{2}$ sheaves of $\mathcal{O}_{X}$-modules and $\mathcal{E}$ ample, then

(1) $f^{*} \mathcal{E}$ is ample (in particular restrictions of ample sheaves are ample)

(2) every quotient of $\mathcal{E}$ is ample

(3) $\mathcal{E}_{1} \oplus \mathcal{E}_{2}$ is ample if and only if $\mathcal{E}_{1}$ and $\mathcal{E}_{2}$ are both ample

Proposition 2.5 ([Laz04, 6.4.17]). Let $C$ be a smooth curve and $\mathcal{E}$ and $\mathcal{F}$ vector bundles on $C$. If $\mathcal{E}$ is ample and there is a homomorphism $\mathcal{E} \rightarrow \mathcal{F}$, surjective outside of finitely many points, then $\mathcal{F}$ is ample.

We need one further result which is, besides Theorem 2.2, the main ingredient for our result:

Theorem 2.6 ([CMSB02, Corollary $0.4(11)]$ ). A uniruled projective complex manifold $X$ of dimension $n$ with a dense open subspace $U$ such that for all $p \in U$ and all rational curves $C$ through $p$ the inequality $-K_{X} . C \geq n+1$ holds, is isomorphic to $\mathbb{P}_{n}$. 


\section{Projective VARieties With AMPLE TANGENT SheaVes}

Now we get to the main result of the paper:

Theorem 3.1. Let $X$ be a normal projective variety over $\mathbb{C}$ of dimension $n$ with ample tangent sheaf $\mathcal{T}_{X}$, then

$$
X \simeq \mathbb{P}_{n}
$$

Before proving the main theorem we have to adapt the results given in Section 2 ,

Theorem 3.2. Let $X$ be a normal projective variety, then there is a desingularisation $\pi: \hat{X} \rightarrow X$ and an $\mathcal{O}_{\hat{X}}$-module homomorphism

$$
f: \pi^{*} \mathcal{T}_{X} \rightarrow \mathcal{T}_{\hat{X}}
$$

that is an isomorphism outside $\pi^{-1}(\operatorname{Sing}(X))$.

Proof. Using Theorem 2.2, we obtain an isomorphism $\mathcal{T}_{X} \rightarrow \pi_{*} \mathcal{T}_{\hat{X}}$ for a suitable resolution $\pi: \hat{X} \rightarrow X$. The map $\pi$ is an isomorphism outside $\pi^{-1}(\operatorname{Sing}(X))$ (one has to retrace the resolution guaranteed by [GK14, Theorem 4.2] to [Kol07, Theorem 3.45] for this property). Pulling back $\mathcal{T}_{X} \rightarrow$ $\pi_{*} \mathcal{T}_{\hat{X}}$ and using the natural morphism $c: \pi^{*} \pi_{*} \mathcal{T}_{\hat{X}} \rightarrow \mathcal{T}_{\hat{X}}$, there is the diagram

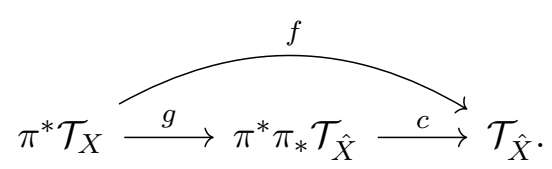

Considering the maps $g$ and $c$, it is easy to check that they, and therefore $f$, are isomorphisms outside $\pi^{-1}(\operatorname{Sing}(X))$.

Remark. The editor pointed out to the author that Kawamata [Kaw85, p. $14]$ made use of the map $f$ as well.

Lemma 3.3. Let $X$ be a normal projective variety of dimension $n$ with ample tangent sheaf $\mathcal{T}_{X}$ and $C \subset X$ a closed curve that intersects $\operatorname{Sing}(X)$ in at most finitely many points. Let $\pi: \hat{X} \rightarrow X$ be a desingularisation as in Theorem 3.2 $\hat{C}$ the strict transform of $C$ and $\eta: \tilde{C} \rightarrow \hat{C}$ the normalisation of $\hat{C}$. Accordingly, there is the following commutative diagram:

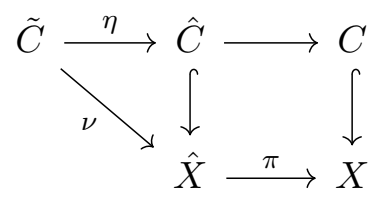


Then $\nu^{*} \mathcal{T}_{\hat{X}}$ is an ample vector bundle and the anticanonical degree $-K_{\hat{X}} . \hat{C}$ is positive. If $\hat{C}$ is a rational curve, $-K_{\hat{X}} . \hat{C} \geq n+1$.

Proof. The choice of $\pi$ yields the map $f: \pi^{*} \mathcal{T}_{X} \rightarrow \mathcal{T}_{\hat{X}}$. Pulling back $f$ via $\nu$ and dividing out the kernel gives

$$
\overline{\nu^{*} f}: \mathcal{A} \hookrightarrow \nu^{*} \mathcal{T}_{\hat{X}}
$$

with $\mathcal{A}:=\nu^{*} \pi^{*} \mathcal{T}_{X} / \operatorname{ker}\left(\nu^{*} f\right)$. The sheaf $\mathcal{A}$ is ample, since $\mathcal{T}_{X}$ is ample, $\pi \circ \nu$ is finite and quotients of ample sheaves are ample again. Moreover $\mathcal{A}$ is locally free of rank $n$ because it is a torsion-free sheaf on a smooth curve, $\pi \circ \nu$ is an isomorphism outside of finitely many points and $\operatorname{ker}\left(\nu^{*} f\right)$ is supported on only finitely many points. Using Proposition 2.5, we deduce that $\nu^{*} \mathcal{T}_{\hat{X}}$ is an ample vector bundle. Because $-K_{\hat{X}} \cdot \hat{C}=\operatorname{deg} \nu^{*} \mathcal{T}_{\hat{X}}$, the anticanonical degree is certainly positive. Since $\nu^{*} \mathcal{T}_{\hat{X}}$ splits on $\mathbb{P}_{1}$ and a direct sum of ample vector bundles is ample only if all summands are ample, we obtain $\nu^{*} \mathcal{T}_{\hat{X}} \simeq \bigoplus_{i=1}^{n} \mathcal{O}_{\mathbb{P}_{1}}\left(a_{i}\right)$ with $a_{i} \geq 1$ for all $i$. The dual of the homomorphism $\nu^{*} \Omega_{\hat{X}}^{1} \rightarrow \Omega_{\tilde{C}}^{1}$ is a non-trivial map $\mathcal{T}_{\mathbb{P}_{1}} \simeq \mathcal{O}_{\mathbb{P}_{1}}(2) \rightarrow \nu^{*} \mathcal{T}_{\hat{X}}$. Thus $a_{i} \geq 2$ for at least one $i$ and we can conclude $-K_{\hat{X}} \cdot \hat{C}=\sum_{i=1}^{n} a_{i} \geq n+1$.

Now we use Lemma 3.3 to show that the assumptions of Theorem 2.6 are fulfilled for $\hat{X}$ and hence $X$ is isomorphic to $\mathbb{P}_{n}$.

Proof of Theorem 3.1 Normal curves are smooth, so we can assume that $n \geq$ 2. Let $\pi: \hat{X} \rightarrow X$ be a desingularisation as in Lemma 3.3 and let $p \in$ $\hat{X} \backslash \pi^{-1}(\operatorname{Sing}(X))$ be any general point outside the exceptional locus.

Since $\hat{X}$ is projective, there is an irreducible curve $\hat{C}$ through $p$. As $\hat{C}$ is the strict transform of a closed curve $C \subset X, K_{\hat{X}} \cdot \hat{C}<0$ according to Lemma 3.3. Therefore $\hat{X}$ is uniruled by [MM86, Theorem 1].

Any rational curve $\hat{C} \subset \hat{X}$ containing $p$ projects to a curve $C$ on $X$. The curve $C$ meets $\operatorname{Sing}(X)$ in at most finitely many points, thus Lemma 3.3 applies and we have the assumptions of Theorem 2.6 fulfilled. So $\hat{X}$ is isomorphic to the projective space $\mathbb{P}_{n}$. Hence $X \simeq \mathbb{P}_{n}$ too. 
Acknowledgement. I want to thank Prof. Dr. Thomas Peternell for his guidance and support and Dr. Patrick Graf for his advice in many occasions, proofreading and especially for hinting me towards [GK14, Theorem 4.2]. In addition I thank Andreas Demleitner and Dr. Florian Schrack for proofreading, their advice and countless conversations.

\section{REFERENCES}

[AKP08] Marian Aprodu, Stefan Kebekus, and Thomas Peternell, Galois coverings and endomorphisms of projective varieties, Math. Z. 260 (2008), no. 2, 431-449. MR 2429621

[Anc82] Vincenzo Ancona, Faisceaux amples sur les espaces analytiques, Trans. Amer. Math. Soc. 274 (1982), no. 1, 89-100. MR 670921

[AW01] Marco Andreatta and Jarosław A. Wiśniewski, On manifolds whose tangent bundle contains an ample subbundle, Invent. Math. 146 (2001), no. 1, 209-217. MR 1859022

[Bal93] Edoardo Ballico, On singular varieties with ample tangent bundle, Indag. Math. (N.S.) 4 (1993), no. 1, 1-10. MR 1213317

[CMSB02] Koji Cho, Yoichi Miyaoka, and N. I. Shepherd-Barron, Characterizations of projective space and applications to complex symplectic manifolds, Higher dimensional birational geometry (Kyoto, 1997), Adv. Stud. Pure Math., vol. 35, Math. Soc. Japan, Tokyo, 2002, pp. 1-88. MR 1929792

[Deb06] Olivier Debarre, On coverings of simple abelian varieties, Bull. Soc. Math. France 134 (2006), no. 2, 253-260. MR 2233707

[GK14] Patrick Graf and Sándor J. Kovács, An optimal extension theorem for 1-forms and the Lipman-Zariski conjecture, Doc. Math. 19 (2014), 815-830. MR 3247804

[GKK10] Daniel Greb, Stefan Kebekus, and Sándor J. Kovács, Extension theorems for differential forms and Bogomolov-Sommese vanishing on log canonical varieties, Compos. Math. 146 (2010), no. 1, 193-219. MR 2581247

[GPR94] H. Grauert, Th. Peternell, and R. Remmert (eds.), Several Complex Variables VII, Springer Berlin Heidelberg, 1994.

[Kaw85] Yujiro Kawamata, Minimal models and the Kodaira dimension of algebraic fiber spaces, J. Reine Angew. Math. 363 (1985), 1-46. MR 814013

[Keb02] Stefan Kebekus, Characterizing the projective space after Cho, Miyaoka and Shepherd-Barron, Complex geometry (Göttingen, 2000), Springer, Berlin, 2002, pp. 147-155. MR 1922103

[Kol07] János Kollár, Lectures on resolution of singularities, Annals of Mathematics Studies, vol. 166, Princeton University Press, Princeton, NJ, 2007. MR 2289519

[Kub70] Kazuji Kubota, Ample sheaves, J. Fac. Sci. Univ. Tokyo Sect. I A Math. 17 (1970), 421-430. MR 0292849

[Laz04] Robert Lazarsfeld, Positivity in algebraic geometry. II, Ergebnisse der Mathematik und ihrer Grenzgebiete. 3. Folge. A Series of Modern Surveys in Mathematics [Results in Mathematics and Related Areas. 3rd Series. A Series of Modern Surveys in Mathematics], vol. 49, Springer-Verlag, Berlin, 2004, Positivity for vector bundles, and multiplier ideals. MR 2095472 
[Liu16] Jie Liu, Characterization of projective spaces and $\mathbb{P}^{r}$-bundles as ample divisors, To appear in Nagoya Mathematical Journal, arXiv:1611.05823, November 2016.

[MM86] Yoichi Miyaoka and Shigefumi Mori, A numerical criterion for uniruledness, Ann. of Math. (2) 124 (1986), no. 1, 65-69. MR 847952

[Mor79] Shigefumi Mori, Projective manifolds with ample tangent bundles, Ann. of Math. (2) 110 (1979), no. 3, 593-606. MR 554387 